\title{
Los retos del docente AICLE
}

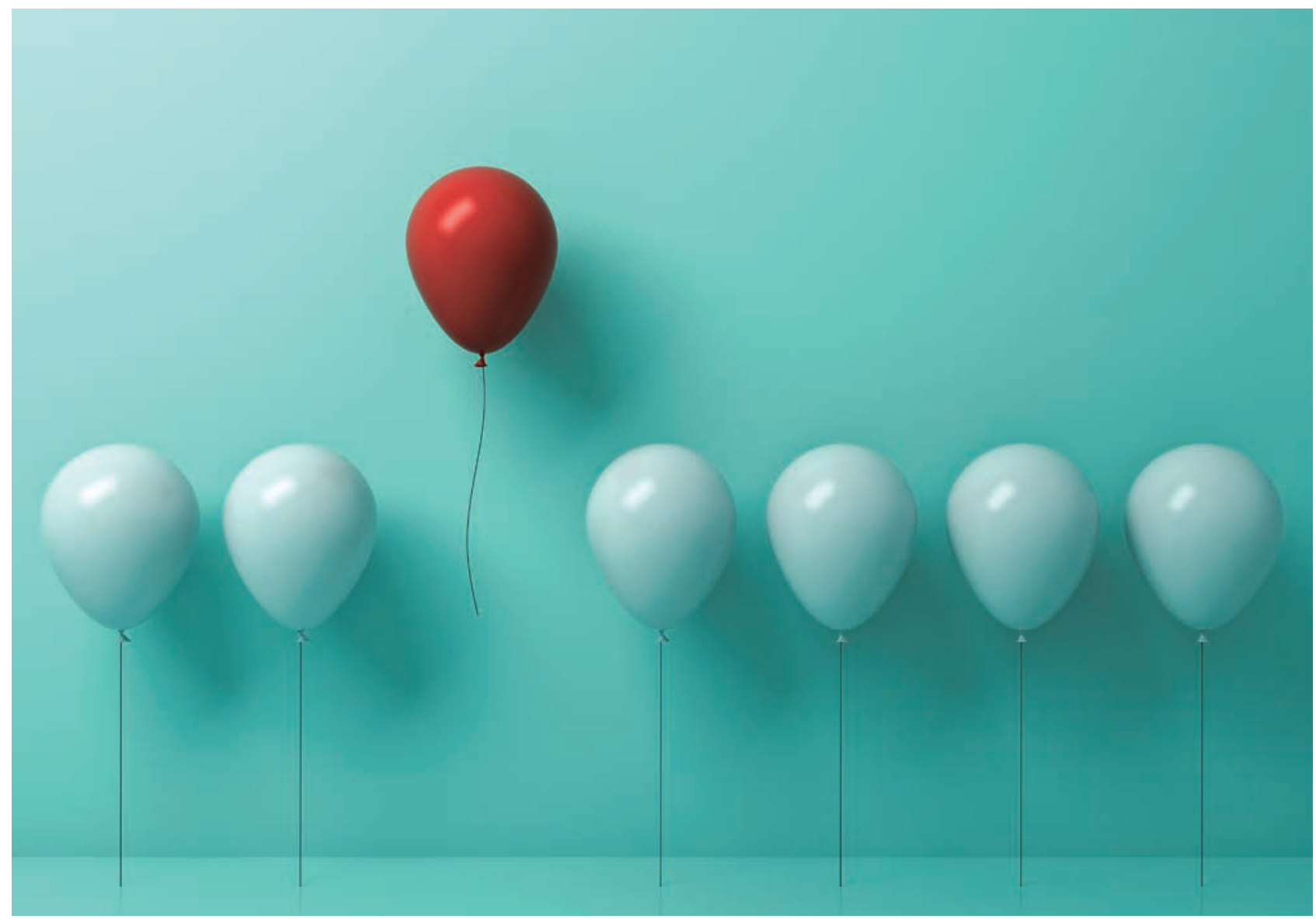

AICLE es mucho más que enseñar en inglés, porque enseñar en un idioma extranjero no es lo mismo que enseñar un idioma extranjero, ni lo mismo que enseñar en la lengua materna. Un buen nivel de inglés de los docentes no es una garantía de una enseñanza bilingüe de

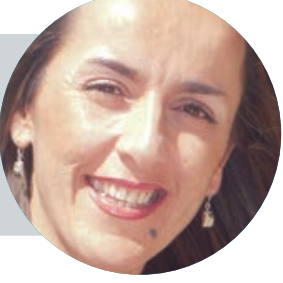
calidad. La formación metodológica en AICLE es fundamental para promover un aprendizaje lingüístico y cognitivo adecuado a las necesidades de cada alumno. Por tanto, es uno de los retos más importantes de la educación bilingüe para garantizar su sostenibilidad a largo plazo. 


\section{El panorama del docente AICLE}

La calidad de los programas bilingües que se desarrollan en España se mide de forma generalizada en base al rendimiento académico de los alumnos. Sin embargo, uno de los factores de calidad de un programa educativo es la adecuada formación de los docentes responsables de su desarrollo en las aulas. Tras casi 20 años de educación bilingüe, es el momento de abrir nuevas vías de evaluación de la calidad de los programas bilingües centradas en su desarrollo y no exclusivamente en los resultados académicos.

Pongamos como ejemplo la Comunidad de Madrid, que presenta su programa con el eslogan: Madrid Comunidad Bilingüe. Este Programa de Educación Bilingüe, que está basado en el AICLE (aprendizaje integrado de contenido y lengua), supone actualmente el $50 \%$ de los centros de infantil, primaria y secundaria madrileños. Sin embargo, desde su implantación, en el año 2004, la calidad del programa se ha medido fundamentalmente en función del rendimiento académico de los alumnos. No se ha medido con la misma intensidad ni con la misma fiabilidad, el impacto que esta innovación ha supuesto para los docentes responsables de su desarrollo en las aulas. Docentes que, para impartir bilingüismo, deben obtener una habilitación previa. Esta habilitación, desde el año 2010, solamente tiene en cuenta el criterio de la competencia de los docentes en la lengua extranjera. ¿Pero qué hay de la competencia metodológica?

\section{La paradoja del docente AICLE}

El contexto actual en el que se desarrollan la formación y la habilitación/acreditación bilingüe pone de manifiesto una gran paradoja en torno a la figura del docente AICLE, que se resume en la figura 1.

La formación inicial del profesorado es insuficiente para dar respuesta a los nuevos modelos educativos bilingües (Jover, Fleta y González, 2016). Basta con mencionar que el nivel exigido en los grados de educación de los profesores generalistas en una mayoría de universidades es solo el B1, según el Marco

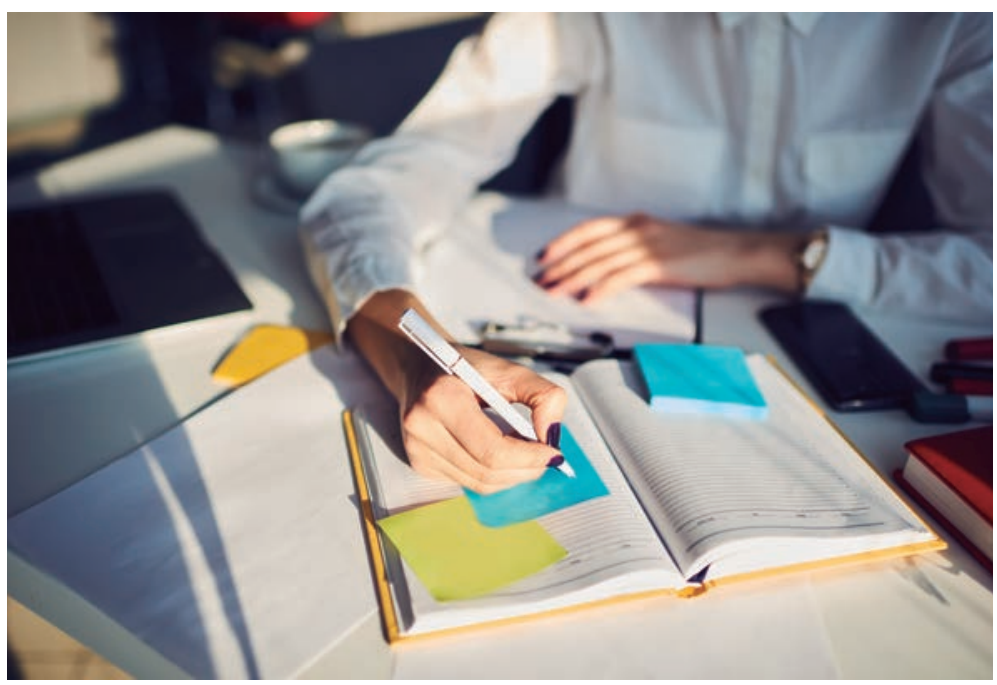

Común Europeo de Referencia (MCER), y en la mención de inglés, el B2. Además, el aprendizaje del contenido pedagógico de la metodología bilingüe se reduce, en la mayoría de los casos, a una asignatura cuatrimestral del grado. Según Morton (2016), la mejora del conocimiento del contenido pedagógico de la metodología bilingüe es uno de los desafíos más importantes que se deben enfrentar en todos los tipos de instrucción basada en el contenido, como es AICLE.

Con respecto a la formación permanente, la mayoría de programas bilingües, aunque ofrecen formación AICLE en sus programas de formación del profesorado,

\section{Figura 1. La paradoja del docente AICLE}

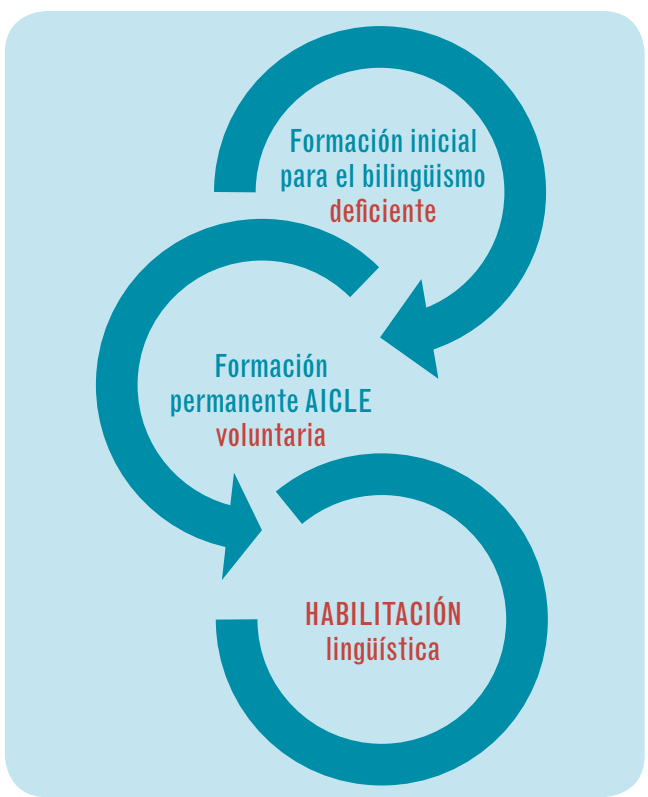




\section{Cuadro 1. Formación del profesorado y requisitos para la educación bilingüe en España}

\begin{tabular}{|c|c|c|c|c|c|c|c|}
\hline CC. AA. & MCER & PIFL/M (1) & Vigencia (2) & Mérito (3) & Incentivo (4) & $\begin{array}{c}\text { Dedicación } \\
\text { horaria (5) }\end{array}$ & $\begin{array}{c}\text { Otras } \\
\text { actuaciones (6) }\end{array}$ \\
\hline Andalucía & B2 & & & Mérito & & Sí & PEE \\
\hline Aragón & B2 & & & & Créditos & Sí & \\
\hline Asturias & B2 & & & & Créditos & Sí & PEE \\
\hline Baleares & B2 & Sí & 3 años & Mérito & Créditos & Sí & \\
\hline Canarias & B2 & Sí & & Certifica & & Sí & \\
\hline Cantabria & B2 & & Indefinida & Certifica & Créditos & Sí & Ayudas \\
\hline Castilla y León & B2 & & & & & Sí & \\
\hline $\begin{array}{l}\text { Castilla La } \\
\text { Mancha }\end{array}$ & B2 & & & Mérito & Económico & Sí & PEE \\
\hline Cataluña & B2 & & & Certifica & Créditos & & PEE \\
\hline C. Valenciana & B2 & Sí & & Mérito & & & \\
\hline Extremadura & B2 & Sí & & Mérito & Ambos & Sí & Ayudas \\
\hline Galicia & B2 & & & Certifica & Créditos & Sí & Ayudas \\
\hline Madrid & C1 & & 3 años & Mérito & Económico & Sí & Ayudas \\
\hline Murcia & B2 & & & Certifica & Créditos & Sí & Ayudas \\
\hline Navarra & $\mathrm{C1}$ & & & & & Sí & \\
\hline País Vasco & B2 & & & & & Sí & Ayudas \\
\hline La Rioja & B2 & Sí & & & Créditos & Sí & \\
\hline Ceuta & B2 & Sí & & & & Sí & \\
\hline Melilla & B2 & Sí & & & & Sí & \\
\hline
\end{tabular}

Leyenda. 1. Plan inicial de formación lingüística/metodológica. 2. Vigencia de la habilitación necesaria para impartir en programas bilingües. 3. Reconocimiento de la participación en el programa como mérito específico en las convocatorias y concursos. 4. Reconocimiento de créditos de formación o complemento de productividad 0 ambos. 5. Dedicación de horas de docencia del profesorado bilingüe al programa bilingüe para programación, formación, elaboración de materiales, etc. 6. Programas educativos europeos que incluyen acciones dirigidas al profesorado o ayudas a la formación

no la exigen como un requisito previo o vinculado al proceso de habilitación/acreditación, relegando esta formación a la voluntad de los docentes. Esto ha dado lugar a un fenómeno muy interesante: la heterogeneidad de perfiles de competencia del docente AICLE.

\section{Las competencias del docente AICLE}

Pero, ¿qué se entiende por competencia AICLE? Son muchos los autores que han definido las competencias de un docente AICLE e incluso han definido un Marco Común Europeo para la Formación del Profesor AICLE (MARSh, Mehisto, Wolf y FrIGOLS, 2010). Se muestra a continuación un resumen de estas competencias.

Cuando se enseña contenido curricular en una lengua extranjera, la competencia pedagógica permite al docente poner en la práctica los principios metodológicos del enfoque AICLE, la competencia científica implica el conocimiento de dichos principios metodológicos, la competencia organizativa es necesaria para integrar de forma efectiva todos los elementos que componen el AICLE, las competencias interpersonal y colaborativa son imprescindibles para generar una trama de relaciones socioafectivas y laborales adecuadas para afrontar el reto que supone el AICLE y la competencia para la reflexión y el desarrollo personal garantizan la actualización profesional. Todas ellas, junto con la competencia lingüística, permiten al docente AICLE desarrollar una enseñanza bilingüe de calidad (Pérez CAÑado, 2018).

\section{Los perfiles del docente AICLE}

Además de estas competencias comunes a todos los docentes AICLE, existen distintos perfiles profesionales que, a su vez, demandan una formación específica 
adecuada a sus necesidades. ¿Cuáles son los perfiles del docente AICLE?

Según los datos recogidos en un estudio realizado con una muestra de 383 docentes habilitados de primaria y secundaria del Programa Bilingüe de la Comunidad de Madrid (Custodio Espinar, 2019), los perfiles del docente AICLE desde el punto de vista de la etapa y las asignaturas que imparten son los que muestran las figuras 3 y 4.

El perfil mayoritario en la etapa primaria es el del docente AICLE habilitado, que imparte conjuntamente las áreas de Ciencias Sociales, Ciencias Naturales y Educación Artística. En la etapa secundaria son los docentes acreditados que imparten el currículo avanzado de Lengua Inglesa y Literatura, seguidos de los profesores AICLE de Ciencias Sociales, Geografía e Historia. Cada uno de estos perfiles requiere una formación pedagógica de la metodología de AICLE propia del área que imparte. ¡No es lo mismo enseñar en inglés las Ciencias Naturales o la Biología que la Historia o la Educación Física!

Salaberri (2010) distingue tres perfiles desde el punto de vista de las necesidades de formación del profesorado.

\ El perfil del profesor de idiomas necesita formación tanto en sus niveles de competencia lingüística como en los enfoques de aprendizaje que promueve en el aula, para poder generar un contexto comunicativo real, propio de un enfoque en el que la lengua se emplea para aprender.

入 El perfil del profesor de contenido necesita mucha formación en su nivel de competencia lingüística, pero, además, formación sobre cómo usar esta lengua como vehículo para el aprendizaje de los contenidos que imparte a través de ella (AICLE).

त El perfil del profesor generalista (que enseña en español) necesita formación para desarrollar una metodología acorde con las anteriores, coordinada tanto en los objetivos como en los contenidos.

Y todos ellos necesitan formación sobre las repercusiones lingüísticas y culturales que supone aprender contenidos
Figura 2. Las competencias del docente AICLE (Pérez C Cañado, 2018)

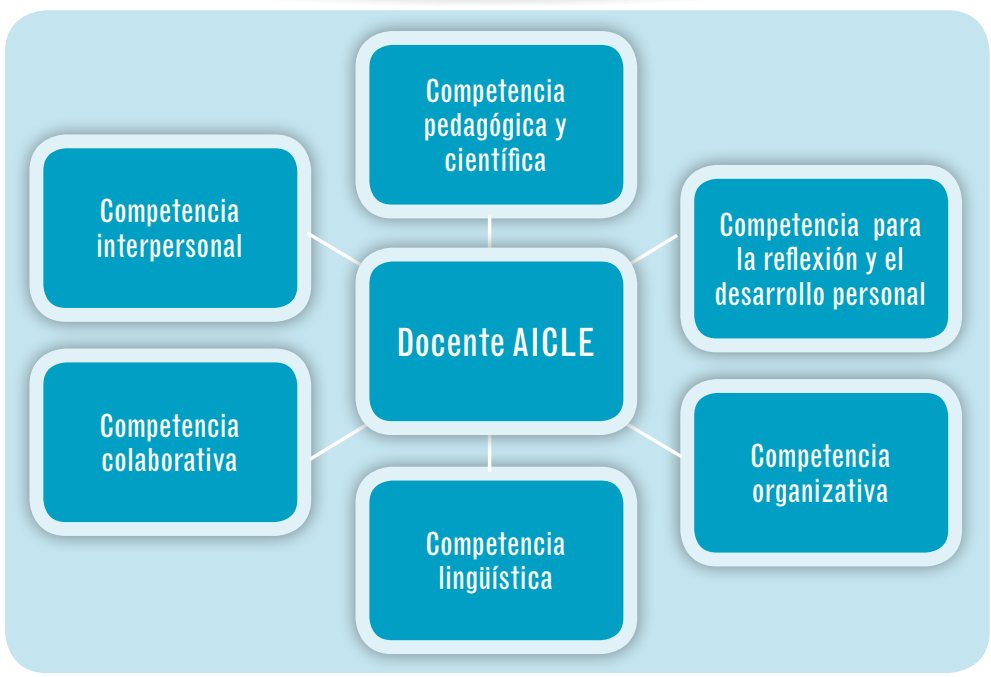

Figura 3. Áreas impartidas en inglés por los docentes de la etapa primaria

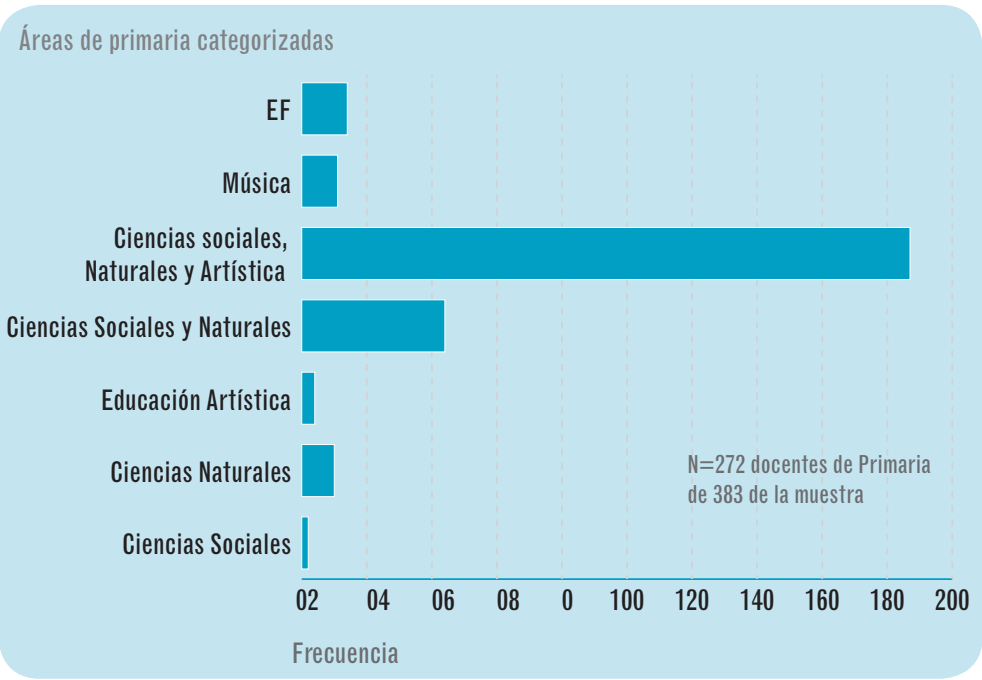

Figura 4. Áreas impartidas en inglés por los docentes de la etapa secundaria

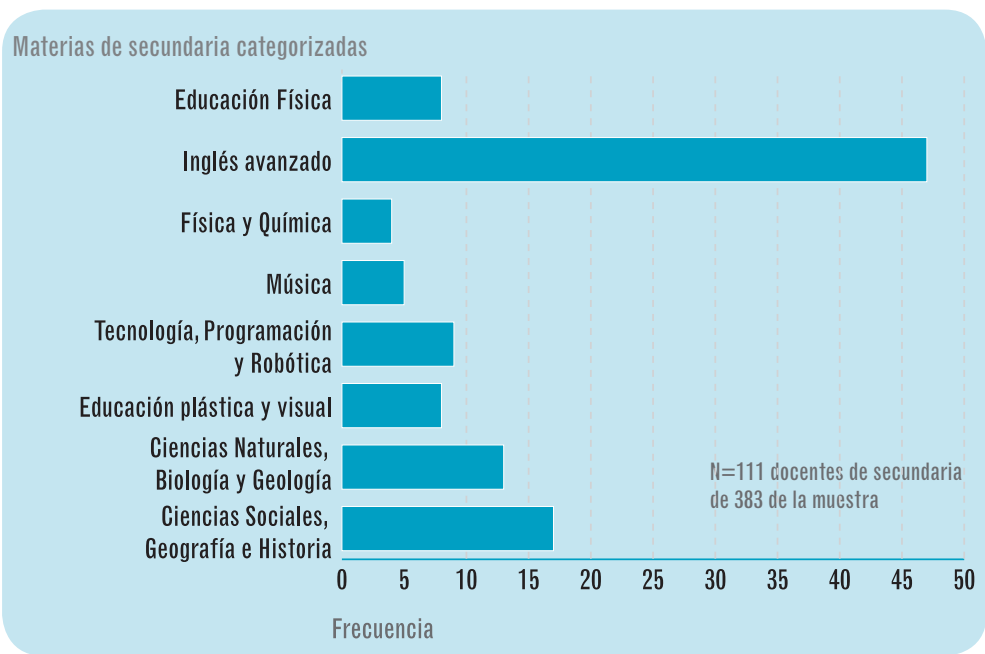


La autoevaluación docente

La programación docente es un factor que determina la calidad de los procesos de enseñanza y aprendizaje. En un contexto tan heterogéneo de perfiles docentes, la evaluación diagnóstica del nivel de integración de los principios metodológicos de AICLE en el estilo de programación docente, mediante herramientas como cuestionarios o checklists, puede servir como referente para el diseño o la elección de cursos de formación. Además, esta evaluación diagnóstica de las necesidades docentes se puede realizar en diferentes niveles: individual, centro, localidad o Comunidad Autónoma.

Una autoevaluación previa a la decisión sobre el tipo de formación que se va a realizar puede resultar muy útil a la hora de orientar el criterio personal para la selección de los cursos de formación que necesita cada docente 0 equipo de docentes, en función de sus perfiles y su nivel de competencia AICLE. Esto es así porque la amplia oferta de formación en AICLE que diseñan las administraciones en los planes de formación permanente del profesorado exige un criterio válido, basado en el conocimiento de las necesidades personales, para la elección de la acción formativa adecuada para cada perfil docente.

\section{El checklist del docente AICLE}

Descriptores de la competencia programación AICLE

Conozco los elementos fundamentales de una programación AICLE: contenido, cognición, comunicación y cultura

Analizo las demandas cognitivas de las tareas que propongo con relación a una taxonomía, por ejemplo, la de Bloom

Planifico las actividades de aprendizaje siguiendo una secuencia lógica de razonamiento y desarrollo cognitivo

Anticipo las demandas del lenguaje que surgen del contenido que se aprende en el aula

Preparo materiales para apoyar el lenguaje en la fase de comprensión, transformación y producción del conocimiento de los alumnos

Diseño tareas de aprendizaje centradas en el alumno

Promuevo el aprendizaje autónomo e independiente de mis alumnos

Conozco los estilos de aprendizaje de mis alumnos y lo tengo en cuenta a la hora de programar

Adapto mi programación a las necesidades cognitivas y linguíisticas de los alumnos, tanto en grupo, como individualmente

Gestiono la participación del auxiliar de conversación en el aula y la planifico previamente

Promuevo el desarrollo de la competencia digital mediante el uso de las TIC como recurso y como contenido de aprendizaje

Planifico y preparo materiales didácticos AICLE (realia, scaffolding, TIC...) en equipo para enriquecer el resultado

Comparto mis programaciones, recursos y materiales con otros compañeros

Desarrollo estrategias de evaluación formativa (durante el proceso) y empleo rúbricas y checklists

Diseño herramientas de evaluación sumativa (exámenes) en las que el lenguaje no es una barrera para evaluar el contenido

Evalúo el contenido, el lenguaje y el proceso de aprendizaje de forma equilibrada y justa

Fuente: elaboración propia a partir del cuestionario CIPMA (cuestionario de integración de los principios metodológicos AICLE). Custooı EsprNar (2019, 536-541).

Elementos fundamentales de AICLE

Metodología AICLE
Recursos de enseñanza AICLE

Evaluación en contextos de AICLE

Comprobar objetivamente las fortalezas y debilidades para programar lecciones de AICLE y reflexionar sobre la mejor opción para mejorarlas es una estrategia que permite adecuar la elección de la formación a las necesidades reales. Existen numerosas herramientas que te permiten evaluar tu competencia docente AICLE. Estas son algunas:

$\searrow$ The TKT (Teaching Knowledge Test) Course. CLIL Module Content and Language Integrated Learning (BentLEY, 2010),

У El registro de control que proponen Dale y Tanner (2012) para comprobar How CLIL are you? Las autoras ofrecen una lista de control con una escala nominal (siempre, frecuentemente, a veces, ocasionalmente, nunca) en la que miden la capacidad del docente para integrar los principios metodológicos que deben estar presentes en una lección AICLE de calidad.

У El CIPMA (cuestionario de integración de los principios metodológicos AICLE), herramienta para la autoevaluación del docente AICLE, dividida en cuatro dimensiones (D1 los elementos fundamentales de AICLE, D2 metodología, D3 recursos y D4 evaluación), que te permite medir el nivel de integración de los principios del enfoque en tu estilo de programación docente (Custodio Espinar, 2019).

\section{Referencias}

BentLey, K. (2010). The TKT (Teaching Knowledge Test) Course. CLIL Module Content and Language Integrated Learning. Cambridge: Cambridge University Press.

Dale, L., \& Tanner, R. (2012). CLIL Activities. A Resource for Subject and Language Teachers, Cambridge: Cambridge University Press. 
en una L2, el desarrollo de estrategias de aprendizaje comunicativo centradas en el alumno, flexibles y que tengan en cuenta la evaluación de la competencia lingüística del alumnado mediante el uso del MCER, para garantizar que la lengua no es un componente invisible del aula AICLE.

De nuevo, según Custodio Espinar (2019), los perfiles desde el punto de vista del modelo de habilitación, la formación AICLE posterior y el nivel de inglés son los que muestra la figura 5.

\section{Los modelos de habilitación son:}

7 Modelo 1, con formación lingüística y AICLE.

7 Modelo 2, con formación AICLE.

入 Modelo 3, sin formación AICLE específica previa.

El tipo de formación posterior a la habilitación incluye los siguientes niveles:

1. Formación posterior AICLE dentro del Plan de la CM.

2. Formación posterior AICLE fuera del Plan de la CM.

3. Formación posterior AICLE dentro y fuera del Plan de la CM.

4. Sin formación AICLE posterior a la habilitación.

El nivel de inglés incluye los niveles B2, C1 y C2 del MCER.

El perfil mayoritario, según estas tres variables, es el de un docente habilitado sin formación AICLE específica, es decir, bajo el criterio de su competencia en lengua extranjera, que no ha recibido formación posterior AICLE, con un nivel de inglés C1 según el MCER. Este perfil confirma la necesidad de revisar el modelo de habilitación para impartir docencia en programas bilingües de forma que se garantice un mínimo de formación metodológica que reduzca las diferencias en los niveles de formación AICLE entre los docentes habilitados.

\section{Conclusión. Los retos del docente AICLE}

Son muchos los autores que afirman que si los planes de formación inicial es-
Decálogo del docente AICLE: formación integral para la enseñanza bilingüe

1. Exige una formación inicial linguística y metodológica en AICLE, porque es una necesidad, un requisito y un derecho de los docentes para poder hacer frente a las demandas de cualquier modelo de enseñanza bilingüe.

2. Defiende una habilitación linguíistica y metodológica como garantía de conocimientos mínimos, que ayudará a reducir la heterogeneidad del perfil de competencias del docente AICLE.

3. Participa en actividades de actualización profesional. En la actualidad hay docentes hiperformados y docentes que no se han formado ni antes ni después de su habilitación. Los equipos directivos y las administraciones educativas deben analizar los factores que inhiben a los docentes de participar en las actividades de formación AICLE y ofrecer soluciones que favorezcan un mayor impacto de las mismas en este colectivo.

4. Colabora y trabaja en equipo como estrategia docente imprescindible para afrontar con garantías el reto de la enseñanza bilinguie. ¡Tú solo no puedes con todo!

5. Cuida y desarrolla tus relaciones interpersonales con alumnos, colegas, auxiliares de conversación, familias, coordinadores y el equipo directivo, porque es fundamental para mantener un nivel de motivación adecuado.

6. Investiga para mejorar tu autoconocimiento, realizar un diagnóstico de tus necesidades de formación y permitir el reconocimiento de tus limitaciones formales y personales como punto de partida para la mejora de tus competencias como docente AICLE y participa del liderazgo de los coordinadores y los equipos directivos orientado a la promoción del desarrollo de las competencias del docente AICLE mediante: el fomento de la formación, (organización de seminarios, participación en programas, cursos, etc.); el intercambio de buenas prácticas y la promoción del trabajo colaborativo (elaboración de programaciones en equipo, accesibilidad a las programaciones y recursos mediante la gestión online de estas tareas, etc.). Todo ello teniendo en cuenta tus necesidades reales de formación.

7. Planifica tu docencia porque la programación docente es un factor determinante de la calidad de los procesos de enseñanza y aprendizaje, especialmente en un contexto de enseñanza AICLE, que implica la integración del desarrollo lingüístico y cognitivo. La programación te permite anticipar las demandas linguiísticas del contenido y reflexionar y planificar adecuadamente la demanda cognitiva de las tareas que propones a tus alumnos.

8. Observa activamente a tus alumnos y a otros colegas, haciendo uso de herramientas que permiten evaluar objetivamente sus competencias y reflexiona sobre ello. Esta es una de las formas más eficaces de aprendizaje y mejora de las competencias propias.

9. Comunica y comparte tus éxitos y tus dificultades con tus alumnos, colegas y familias. El conocimiento de los retos que afrontan los docentes AICLE en el aula pone en valor el esfuerzo y el compromiso que estos manifiestan con un modelo educativo innovador, que debe ser conocido por todos los miembros de la comunidad educativa.

10. Reconoce tu labor como docente AICLE. El bilingüismo es mucho más que enseñar en inglés, es un modelo de enseñanza que demanda un gran esfuerzo linguiístico y cognitivo tanto a los alumnos como a los docentes. 
Figura 5. Perfil del docente AICLE desde el punto de vista del modelo de habilitación, la formación AICLE posterior y el nivel de inglés

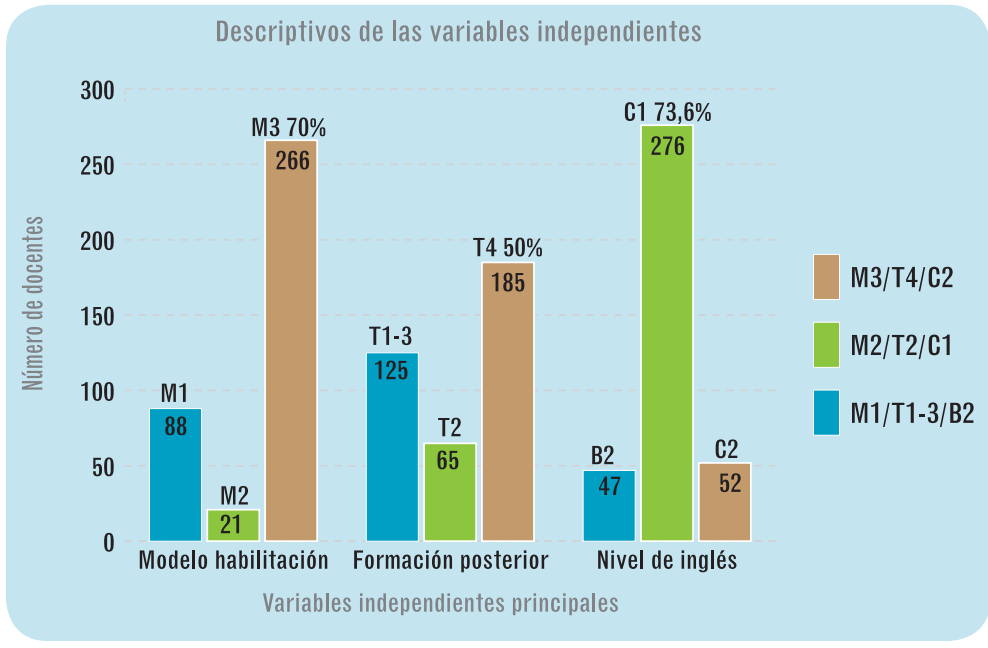

MADRID, 2014), con la consiguiente ventaja que significaría para aumentar la calidad y cantidad de la oferta formativa, pero sobre todo, para orientar la política de formación docente hacia nuevos campos

\section{(D) DARA SABER MÂS}

Custodio Espinar, M. (2019). Los principios metodológicos A/CLE (aprendizaje integrado de contenido y lengua). Colección Tesis doctorales cum laude. Serie P (Pedagogía), N. ${ }^{\circ}$ 10. Madrid: Fundación Universitaria Española.

Morton, T. (2016). Conceptualizing and investigating teachers' knowledge for integrating content and language in content-based instruction. Journal of Immersion and Content-Based Language Education, 4(2), 144-167. doi: https://doi.org/10.1075/jicb.4.2.01mor

Pérez Cañado (2018). Innovations and Challenges in CLIL Teacher Training. Theory Into Practice, 57(3), 1-10. doi: https://doi.org/10.1 080/00405841.2018.1492238

\section{Referencias}

Jover, G., FletA, T., y GonZÁlez, R. (2016). La Formación Inicial de los Maestros de Educación Primaria en el Contexto de la Enseñanza Bilingüe en Lengua Extranjera. Bordón, Revista de Pedagogía, 68(2), 121-135.

Madrid MANRIQUe, M., y MAdRID Fernández, D. (2014). La formación inicial del profesorado para la educación bilingüe. Granada: Universidad de Granada.

Marsh, D., Mehisto, P., WolfF, D., \& Frigols, M. J. (2010). European Framework for CLIL Teacher Education: A framework for the professional development of CLIL teachers. Graz: European Centre for Modern Languages.

SalABerRI RAmIRO, S. (2010). Teacher Training Programmes for CLIL in Andalusia. In D. Lasagabaster \& Y. Ruiz de Zarobe (eds.), CLIL in Spain: Implementation, Results and Teacher Training, (pp. 140-161). Newcastle upon Tyne: Cambridge Scholars Publishing. de investigación que garanticen un mayor impacto de estos planes en el conjunto de la comunidad docente de AICLE.

El modelo actual que habilita a los docentes según el criterio de la competencia lingüística genera diferentes perfiles de docentes habilitados: docentes que han recibido formación AICLE antes y después de su habilitación y docentes que no han recibido ninguna formación AICLE, ni antes, ni después de ser habilitados (Custodio Espinar, 2019).

Por todo ello, se puede concluir que existen importantes retos en torno a la figura del docente AICLE que se deben afrontar como garantía de la sostenibilidad a largo plazo de los programas de educación bilingüe que se desarrollan en España.

Reto 1. Se debe diseñar la formación del docente AICLE desde una perspectiva integral de la "competencia docente AICLE". La competencia lingüística es condición necesaria, pero no suficiente.

Reto 2. La definición de las competencias y la identificación de los perfiles de competencia puede y debe orientar el diseño de los futuros planes de formación inicial y permanente del profesorado AICLE, para favorecer su optimización en tiempo, esfuerzo y recursos, y garantizar un mayor impacto en el conjunto de este colectivo docente.

Reto 3. La habilitación debe contemplar la competencia AICLE y no exclusivamente la competencia lingüística que, aunque imprescindible, no es suficiente para garantizar una enseñanza bilingüe de calidad $•$

\section{(a) HEMOS HABLAO Df \\ Profesorado; formación inicial; formación permanente; competencias; programación AICLE.}

Este artículo fue solicitado por PADRES Y MAESTROS en diciembre de 2018, revisado y aceptado en marzo de 2019. 